\title{
FLOW CYTOMETRIC ANALYSIS OF ERYTHROPOIETIC ABNORMALITY: CHANGES IN THE CELL MATURITY INDEX OF RETICULOCYTES AND RETIC DISTRIBUTION INDEX ARE USEFUL AS INDICATORS OF ERYTHROPOIETIC TOXICITY IN NON-CLINICAL STUDIES
}

\author{
Satoko KAKIUCHI, Mitsuru KOBAYSHI, Yoshihide SATOMI, Daishiro MIURA, \\ Yoshinori KASAHARA and Shiro KONDO \\ Pharmacology and Safety Research Department, \\ Pharmaceutical Development Research Laboratories TEIJIN Pharma Limited, \\ 4-3-2 Asahigaoka, Hino, Tokyo 191-8512, Japan
}

(Received December 10, 2005; Accepted January 22, 2006)

\begin{abstract}
We performed a flow cytometric (FCM) analysis of the maturity of reticulocytes using peripheral blood obtained from rats administered 5 -fluorouracil (5-FU) at 10,50 and $100 \mathrm{mg} / \mathrm{kg}$ or acethylphenyl hydrazine (APHZ) at 1 and $3 \mathrm{mg} / \mathrm{kg}$ to clarify whether the FCM method is useful for assessing toxicity. In the 5-FU-administered rats, a decrease and recovery of the immature reticulocyte fraction (Cell Maturity Index, CMI; Retic Distribution Index, RDI) was observed more rapidly (several days prior to changes in the reticulocyte ratio), and sensitively regarding dose-dependency (clear changes were observed at $10 \mathrm{mg} / \mathrm{kg}$, whereas the reticulocyte ratio was only slightly affected). In addition, there was good agreement between the microscopic results obtained by counting Heilmyer's reticulocyte maturation groups, especially for type I and II, and CMI/RDI assessed by the FCM method after the administration of 50 and $100 \mathrm{mg} / \mathrm{kg}$ of $5-\mathrm{FU}$, the dose at which clear changes were obtained with the microscopic method. In the APHZ-administered rats, a dose-dependent increase in CMI/RDI coinciding with the enhancement of reticulocyte production was observed. The results suggested that the automated FCM method could be a useful and valuable tool to assess and predict impairments of erythropoiesis, especially for CMI and RDI, and could help in the diagnosis of hematological disorders in experimental animals.
\end{abstract}

KEY WORDS: Flow cytometry, Reticulocyte, Cell Maturity Index, Retic Distribution Index, 5-Fluorouracil, Acethylphenyl hydrazine

\section{INTRODUCTION}

The enumeration of reticulocytes in peripheral blood is an important hematological measure of erythrocyte production and a valuable tool for obtaining information about erythropoiesis. For example, the reticulocyte count is clinically important in the evaluation of patients with anemia, helping with the classification of the disease: hypoplastic anemia is caused by decreased production of erythrocytes in the bone marrow resulting in a decrease in the number of reticulocytes in peripheral blood; hyperplastic anemia is caused by increased loss or dysfunction of erythrocytes resulting in an increase in the number of reticulocytes (Koepke, 1991). In non-clinical toxicity studies, an anemia caused by chemicals is also an important toxicity to be assessed.

For many years, reticulocyte numbers were quantified manually by means of a microscope after staining with a supravital dye, new methylene blue (Brecher and Schneiderman, 1950). The early (immature) reticulocyte contains an extensive ribosomal system for protein synthesis, mitochondria, a centriole, and remnants of Golgi bodies. The reticulum is defined as the precipita-

Correspondence: Daishiro MIURA (E-mail: d.miura@teijin.co.jp) 
tion and aggregation of these structures (particularly ribonucleoprotein) by supravital dye techniques. Heilmeyer (1932) divided reticulocytes into four groups based on morphology and the intracellular distribution of the reticulum. Heilmyer's grouping has been considered to reflect the degree of maturity of the reticulocyte, and is used for diagnosis. However, this manual method is tedious and time-consuming, as 1000 erythrocytes must be counted, and are subject to interpretive errors.

To improve the performance of reticulocyte measurements in routine laboratory testing, an automated reticulocyte counting method using flow cytometry (FCM) combined with fluorescent-staining (Thiazoleorange or Auramine-O) has been developed (Koepke, 1991; Siekmeier et al., 2000). Siekmeier et al. (2000) reported that the automated method allows the efficient and reliable determination of reticulocyte counts under routine clinical conditions. The advantage of the FCMbased method is that the amount of fluorescence in a particular cell is proportional to the amount of RNA present, which in turn relates to the maturity of the reticulocyte (Koepke, 1991). As for its clinical diagnostic value, the automated method was reported to provide a useful early measurement of erythropoiesis (Davis and Bigelow, 1990; Greinix et al., 1994; Spanish multicentric study group for hematopoietic recovery, 1994; Dalal et al., 1996). From a physiopathological standpoint, in anemic patients, the automated FCM method was also reported to be useful for helping to distinguish hematological disorders (Davis et al., 1993; Watanabe et al., 1994; d'Onofrio et al., 1996). However, regarding non-clinical toxicity studies, the evaluation of reticulocyte maturity using the total reticulocyte count obtained by the FCM method has yet to be assessed.

In the present study, we performed FCM analysis of the maturity of reticulocytes using peripheral blood obtained from rats administered 5-fluorouracil (5-FU) or acethylphenyl hydrazine (APHZ) to clarify whether the FCM method can be used to assess toxicity, as well as for clinical applications. The results suggested that the automated method is a useful and valuable tool for assessing and predicting impairments of erythropoiesis, and could help in the diagnosis of hematological disorders in experimental animals, as well as clinical diagnosis.

\section{MATERIALS AND METHODS}

\section{Chemicals}

5-FU was purchased from Kyowa-Hakko Kogyo (50 mg/ml vial, Tokyo), APHZ, from Wako Pure
Chemical Industries (Osaka, Japan), and the new methylene blue solution, Wright solution, May-Grünwald solution and Giemsa solution, from Muto Pure Chemicals Co. Ltd. (Tokyo, Japan).

\section{Animals and animal housing \\ Six-week-old male Crl:CD(SD) rats were obtained from Charles River Japan Inc. (Tsukuba, Japan) and housed in suspended metal cages. The ani- mals were acclimated for 1 week prior to examination. The age of the animals at administration was 7 weeks. Solid food (CRF-1, Oriental Yeast Inc., Tokyo, Japan) was freely provided in a stainless steel food container. Sterilized well water was supplied ad libitum by an automatic watering device. The animal room was kept at $24 \pm 1^{\circ} \mathrm{C}$ and $55 \pm 15 \%$ relative humidity. The room air was ventilated 15 times per hour and a $12 \mathrm{hr} / 12 \mathrm{hr}$ light-dark cycle (lighting, 6:00-18:00) was imposed. All experimental procedures involving animals and related protocols were approved by the Committee on Animal Care of the TEIJIN Institute for Biomedical Research.}

\section{Administration of chemicals and blood sampling \\ 5-FU was administered intravenously to rats through the tail. Physiological saline was used as a vehicle, and the volume administered was $2 \mathrm{ml} / \mathrm{kg}$. APHZ was administered orally by gavage. Distilled water was used as a vehicle, and the volume adminis- tered was $5 \mathrm{ml} / \mathrm{kg}$. Peripheral blood (approximately 1 $\mathrm{ml} /$ animal) was collected from the carotid artery under anesthesia with pentbarbital using EDTA-2K as an anti-coagulant.}

\section{Flow cytometric analysis of reticulocytes}

A reticONE ${ }^{\mathrm{TM}}$ reagent kit (Beckman Coulter, Fullerton, CA) and an EPICS XL-MCL (Beckman Coulter) equipped with COULTER reticONE system software were used to identify and enumerate reticulocytes. The kit contains a Retic-STAT ${ }^{\mathrm{TM}}$ (Acridineorange dye) reagent and Retic-CAL ${ }^{\mathrm{TM}}$ biological calibrator. The Acridine-orange dye stains cellular DNA and RNA: it excites at $488 \mathrm{~nm}$ and emits at $500-550 \mathrm{~nm}$ on binding to DNA, and 630-700 $\mathrm{nm}$ on binding to RNA. The Retic-CAL biological calibrator contains stabilized human erythrocytes in an isotonic, bacteriostatic medium. One $\mathrm{ml}$ of Retic-STAT ${ }^{\mathrm{TM}}$ reagent was added to $2 \mu \mathrm{l}$ of the blood to be examined, vortexed gently, and incubated for $20 \mathrm{~min}$ at room temperature protected from light. After incubation, an automated analysis was carried out with the EPICS XL-MCL. 
Flow cytometric analysis of erythropoietic abnormality.

Briefly, the erythrocyte population was enclosed in a gate in the forward scattered (FS) and side scattered (SS) light-cytogram. Regions of mature erythrocyes or reticulocytes were defined based on the RNA content in each cell by analyzing the cellular fluorescent intensity after binding of the dye to RNA. Optimization of the erythrocyte gate, the identification of reticulocytes, and calculation of the Cell Maturity Index (CMI) and the Retic Distribution Index (RDI) were performed automatically by the analysis algorithm of reticONE system software. Forty thousand erythrocytes per sample were analyzed. Flow-Check ${ }^{\mathrm{TM}}$ (Beckman Coulter) was used for verification of the flow cytometer's optical alignment and fluidics system. RETIC-CHEX ${ }^{\circledR}$ (Streck Laboratories, Inc., Omaha, NE) was used as a control for evaluating the accuracy and precision of the automated system.

\section{Manual counting of reticulocytes}

The method described by Brecher and Schneiderman (1950) was performed. The new methylene blue solution was mixed with an equal volume of peripheral blood, and incubated for 15 to $20 \mathrm{~min}$ at room temperature. Slides were prepared using a cytocentrifuge (HITACHI 806-0400; HITACHI Science Systems, Ltd., Hitachinaka, Ibaragi, Japan), and stained with Wright solution. The microscopic observation was performed using an ocular micrometer disk as described by Brecher, and the reticulocyte ratio and Heilmyer's maturation groups were determined.

\section{Blood chemical and hematological examinations and a bone marrow examination/myelogram in APHZ- administered rats}

For the APHZ-administered rats, peripheral blood and bone marrow were collected after two weeks of repeated oral administration. For the hematological examination, peripheral blood was collected from the carotid artery using EDTA-2K as an anti-coagulant. Erythrocyte count, hemoglobin and hematocrit were examined using a Sysmex E-5000 (Sysmex Corporation, Kobe, Japan). MCHC, MCH and MCV were calculated based on the values of the erythrocyte count, hemoglobin and hematocrit. For the blood chemical examination, serum was collected, and then total bilirubin and serum iron $(\mathrm{Fe})$ were examined using a HITACHI 7250 (HITACHI Science Systems, Ltd.). Bone marrow was collected from the femur and suspended in fetal bovine serum, and then smears were prepared by the wedge method. Smears were stained with May-Grünwald/Giemsa solution, and micro- scopic observations were made. The enumeration of bone marrow nucleated cells was done by the method described by Kakiuchi et al. (2004).

\section{Statistical analysis}

Dunnett's test (Dunnett, 1964) was performed. Levels of significance were $\mathrm{p}<0.05, \mathrm{p}<0.01$, and $\mathrm{p}<0.001$. Analysis was performed using EXSAS software (Ver.7.10).

\section{RESULTS}

The cytograms and histogram obtained by FCM analysis of reticulocytes with the reticONE system

The cytograms and histogram in Fig. 1 represent typical results for the reticONE system after analyzing peripheral blood obtained from an untreated normal rat. First, erythrocytes were gated in region A by analyzing the FS and SS light (Cytogram 1). Cytogram 2 shows the result of determining the DNA or RNA content of cells in region A. Mature erythrocyes are in region D, and reticulocytes are in region E (Cytogram 3). Histogram 1 shows the distribution-pattern of the RNA content of each reticulocyte fraction in region E expressed as the fluorescent intensity after the dye binds to RNA. The Cell Maturity Index (CMI) is the ratio of reticulocytes that are at least 10 times brighter than the dimmest reticulocytes. The $\mathrm{CMI}$ is calculated as follows:

$$
\begin{array}{cc}
\mathrm{CMI}=\begin{array}{c}
\mathrm{c}=\mathrm{m} \\
\begin{array}{c}
\sum_{\mathrm{n}}(\mathrm{c}) \\
\mathrm{c}=10 \mathrm{p}
\end{array}
\end{array} & \begin{array}{l}
\mathrm{p}=\text { channel } \\
\mathrm{c}=\mathrm{m}
\end{array} \\
\begin{array}{c}
\sum_{\mathrm{n}}(\mathrm{c}) \\
\mathrm{c}=\mathrm{p}
\end{array} & \begin{array}{c}
\mathrm{m} \text { maximum channel of RDI region } * \\
\text { histogram }
\end{array} \\
& (* \text { population of channel } \mathrm{c} \\
& (* \text { region } \mathrm{G} \text { in Histogram } 1)
\end{array}
$$

The Retic Distribution Index (RDI) sums a value from each channel by weighing the number of events in the channel by how far that channel is from the peak. Channels with large numbers of counts far to the right of the peak have more weight. This total sum is then normalized by dividing it by the total number of reticulocytes weighed with the maximum peak-to-channel distance. The RDI is calculated as follows:

$$
\begin{aligned}
& \mathrm{c}=\text { channel } \\
& \mathrm{p}=\text { peak channel of RDI } \\
& \text { region* } \\
& \mathrm{RDI}=\frac{\sum_{\mathrm{n}}(\mathrm{c}) \cdot(\mathrm{c}-\mathrm{p})}{\mathrm{c}=\mathrm{p}} \\
& \mathrm{m}=\text { maximum channel of } \\
& \text { the histogram } \\
& \mathrm{n}=\text { population of channel } \mathrm{c} \\
& \text { (* : region G in Histogram 1) }
\end{aligned}
$$



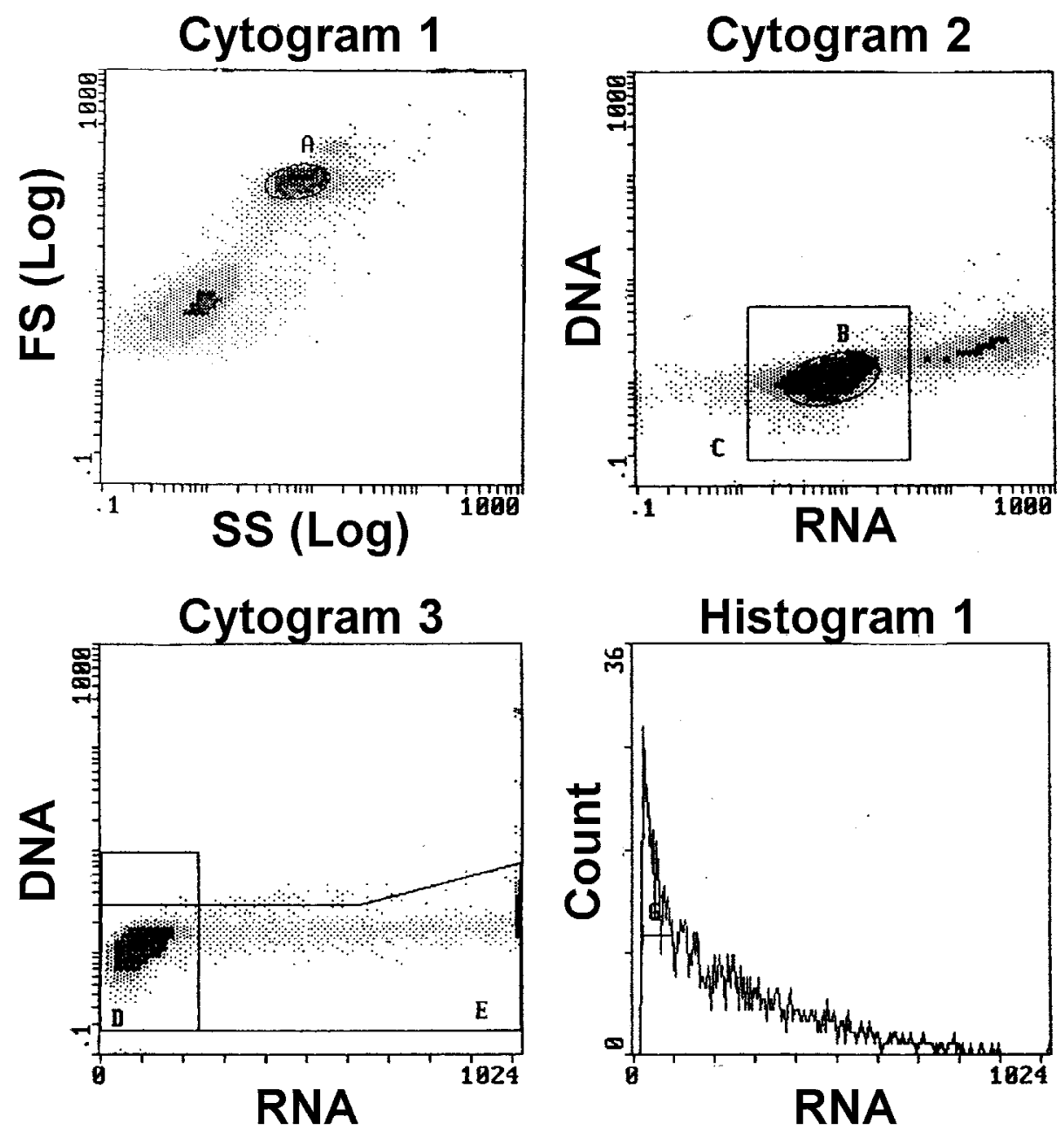

Fig. 1. Cytograms and histogram obtained by EPICS XL-MCL equipped with a reticONE system for examining reticulocyte measurements.

The cytograms and histogram represent typical results for the reticONE system. Peripheral blood obtained from an untreated normal rat was analyzed. Cytogram 1: Scatter plot shows the erythrocyte population enclosed in gate "A". This region is first autogated by analyzing the forward scattered (FS) and side scattered (SS) light and then expanded to include the entire erythrocyte population cluster; Cytogram 2: Two-parameter plots used to define the mature erythrocyte versus reticulocyte populations by analyzing the DNA or RNA content of cells in region "A". The majority of the mature cells are located in an elliptical region "B" contained within a rectilinear region " $C$ ". Region "B" designated as the region over the target population is automatically adjusted by the algorithm based on criteria of the reticONE system. Region " $\mathrm{C}$ " is designated a "validity prime", which signals the software to perform a comparison check to ensure that the peak channel of the population cytogram is within the region; Cytogram 3: Region " $\mathrm{D}$ " is the rectilinear box containing the mature erythrocyte population. Region " $\mathrm{E}$ " is the odd-shaped rectilinear area that begins at the right margin of " $\mathrm{D}$ " and extends to the far right edge of the cytogram; this region represents the reticulocytes. The reticulocyte ratio was calculated by dividing the number of cells in region " $E$ " by the total number of cells in region " $D$ " plus "E"; Histogram 1: The pattern of the distribution of RNA content in cells in region "E". 
Flow cytometric analysis of erythropoietic abnormality.

Fig. 2 shows a cytogram and histogram representing typical results obtained by analyzing peripheral blood which showed a decrease (Fig. 2A) or increase (Fig. 2B) in the reticulocyte ratio, CMI and RDI. In Fig. 2A, cells in region E (the left cytogram) markedly decreased in number, and the reticulocyte ratio was approximately one-tenth that in Fig.1. Cells with a high RNA-content were not observed (the right histogram), and the CMI and RDI were zero. In Fig. $2 \mathrm{~B}$, an increase in the number of cells in region $\mathrm{E}$ was observed, and cells with a high RNA-content clearly increased as compared with Histogram 1 in Fig. 1. The reticulocyte ratio was increased approximately 1.5 folds, and CMI and RDI were increased approximately twofold, as compared with values in Fig. 1.

(A)
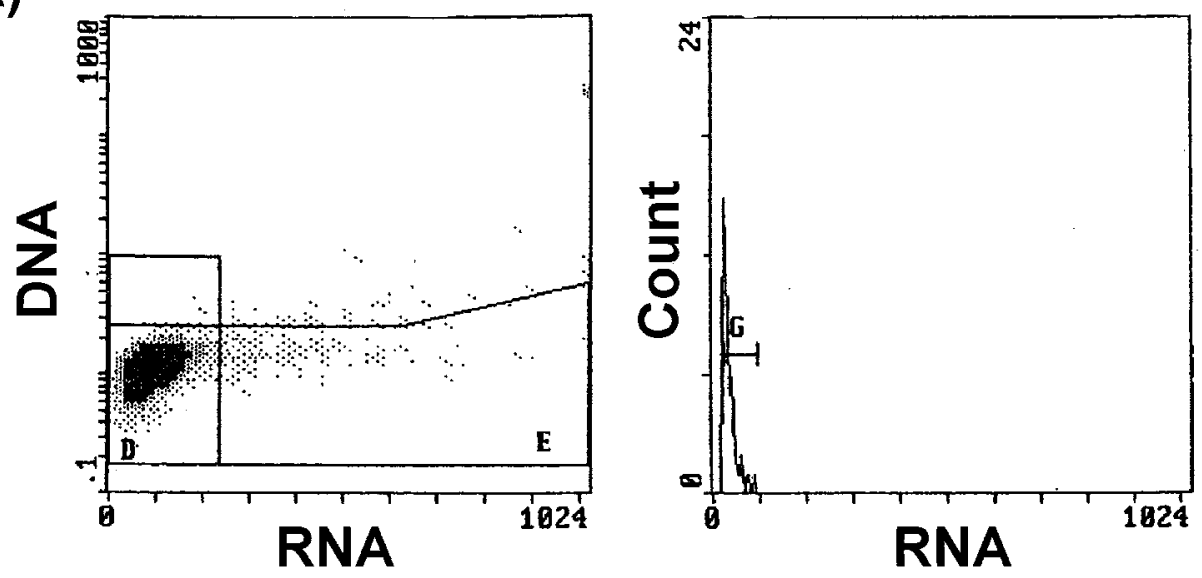

(B)
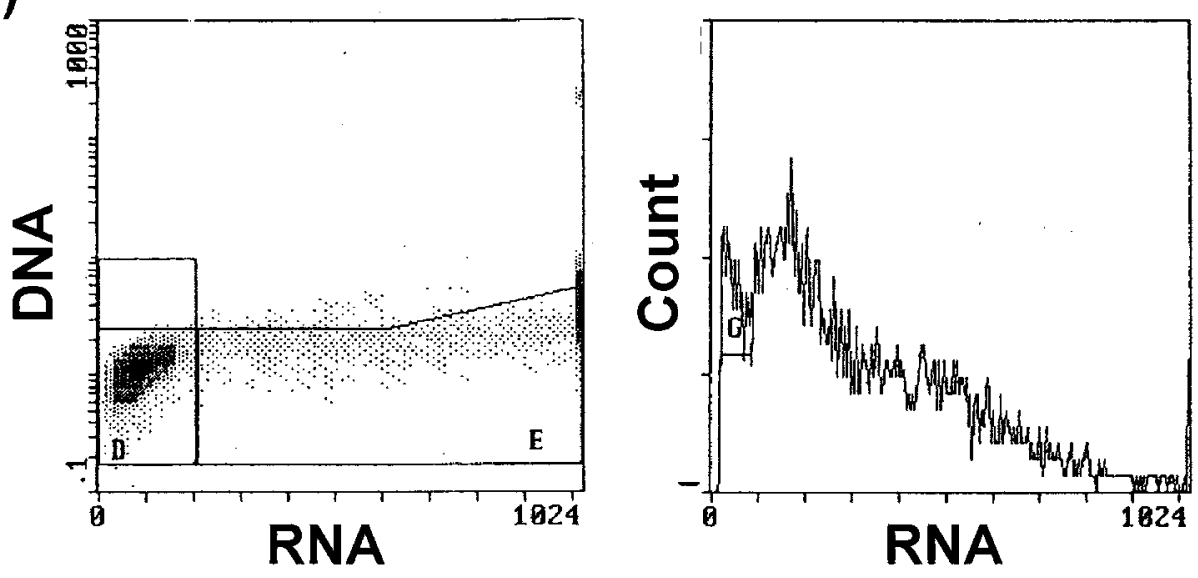

Fig. 2. Cytogram and histogram showing a decreased or increased reticulocyte ratio, Cell Maturity Index and Retic Distribution Index.

Peripheral blood was collected 4 days (A) or 7 days (B) after the single intravenous administration of 5-fluorouracil at $50 \mathrm{mg} / \mathrm{kg}$, and a reticulocyte analysis was carried out using EPICS XL-MCL. Regions "D" and "E" in the left cytogram are designated the same as in Fig. 1. (A) Reticulocyte population (region "E") decreased (reticulocyte ratio $=0.66 \%$ ), and CMI or RDI was zero; (B) Reticulocyte population increased (reticulocyte ratio $=15.56 \%$ ). Cells with a high RNA-content also increased, and CMI and RDI were $50.54 \%$ or 28.63 , respectively. 
Changes in the maturity of reticulocytes after administration of 5-FU

Fig. 3 shows the changes in the reticulocyte ratio, CMI and RDI examined by EPICS XL-MCL using

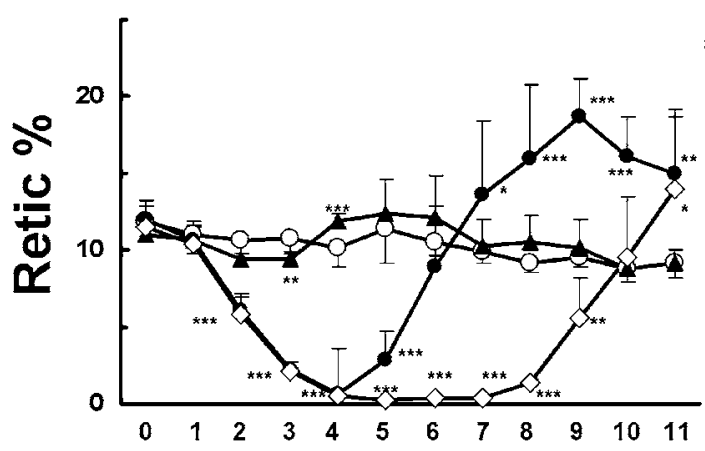

(A)

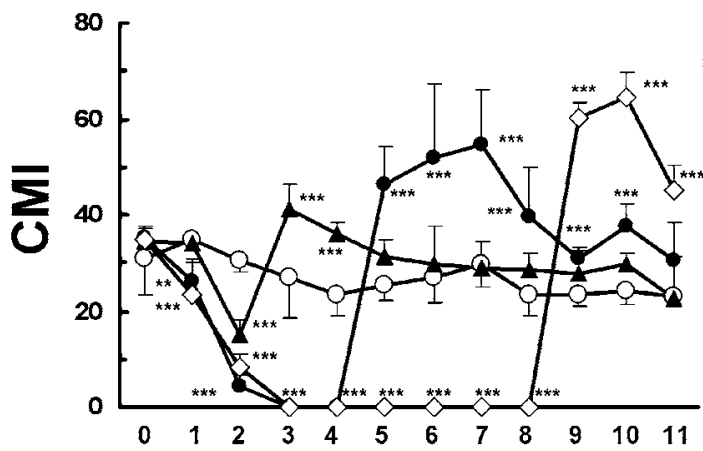

(B)

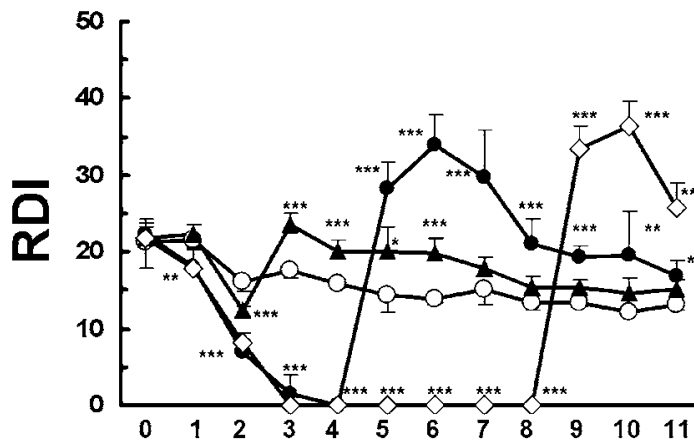

\section{Day}

Fig. 3. Results of reticulocyte ratio (Retic\%), Cell Maturity Index (CMI) and Retic Distribution Index (RDI) of peripheral blood collected from 5-fluorouraciladministered rats.

Peripheral blood was collected 1-11 days after the administration (on Day 0) of 5-fluorouracil at $10(\boldsymbol{\Delta})$, $50(\bullet)$ and $100(\diamond) \mathrm{mg} / \mathrm{kg}$. Control animals $(\mathrm{O})$ were administered physiological saline. Retic\%, CMI and RDI were analyzed using EPICS XL-MCL. Values are the means and $\mathrm{SD}(\mathrm{n}=6) . * \mathrm{p}<0.05, * * \mathrm{p}<0.01$, $* * * \mathrm{p}<0.001$ peripheral blood obtained from rats after a single intravenous administration of $5-\mathrm{FU}$ at 10,50 and $100 \mathrm{mg} /$ $\mathrm{kg}$. As for the ratio of reticulocytes (Fig. 3A), slight changes were observed on Days 3 and 4 when $10 \mathrm{mg} /$ $\mathrm{kg}$ was administered. At $50 \mathrm{mg} / \mathrm{kg}$ of 5 -FU, the reticulocyte ratio (Retic\%) began decreasing on Day 2 and showed a trough on Day 4, but then recovered after Day 5 reaching the control level on Day 6. Interestingly, a high ratio as compared with the control group was observed after Day 7 with a peak on Day 9. A decrease in Retic\% was also observed on Day 2 and thereafter in the $100 \mathrm{mg} / \mathrm{kg}$ administered group, with a trough between Days 4 and 8, and then a recovery after Day 9 and the control level reached on Day 10. As for the CMI (Fig. 3B), a clear decrease was observed on Day 2 at $10 \mathrm{mg} / \mathrm{kg}$. A high CMI as compared with the control value was observed between Days 3 and 4 at this dose. At $50 \mathrm{mg} / \mathrm{kg}$, the CMI began decreasing on Day 1 and thereafter showed a trough between Days 3 and 4 . Then recovery was observed, and a higher value as compared with the control group was observed after Day 5 with a peak on Day 7. There was a return to the control level on Day 11. A marked decrease was also observed on Day 2 and thereafter in the $100 \mathrm{mg} / \mathrm{kg}$ administered group, with a trough between Days 3 and 8 , and then recovery. A higher value as compared with the control group was observed on Day 9 and thereafter with a peak on Day 10. The pattern of change in the RDI after the administration of 5-FU (Fig. 3C) was almost the same as that in the CMI.

Fig. 4 shows the results of the manual counting of reticulocytes using peripheral blood derived from the same animals on which the FCM assay (Fig. 3) was carried out. No statistical changes were observed after the administration of $10 \mathrm{mg} / \mathrm{kg}$ of 5 -FU (data not shown). The ratio of reticulocytes was markedly decreased on Day 4 for both 50 and $100 \mathrm{mg} / \mathrm{kg}$ (Fig. $4 \mathrm{~A})$. A recovery or higher value as compared with the control was observed on Days 7 and 10 at $50 \mathrm{mg} / \mathrm{kg}$. As for the $100 \mathrm{mg} / \mathrm{kg}$ administered animals, there was a marked decrease until Day 7, then a recovery on Day 10. The results of Heilmyer's reticulocyte maturation grouping (type I-IV) are shown in Figs. 4B-4E. At 50 $\mathrm{mg} / \mathrm{kg}$, a marked increase was observed on Days 7 and 10 in the type I and II reticulocytes. A recovery from the decrease was observed on Day 10 in the type I and II reticulocytes at $100 \mathrm{mg} / \mathrm{kg}$, and a marked increase in the type I reticulocytes was also observed on Day 10 at $50 \mathrm{mg} / \mathrm{kg}$. While a recovery from the decrease was observed on Day 7 and thereafter $(50 \mathrm{mg} / \mathrm{kg}$ ) or Day 10 $(100 \mathrm{mg} / \mathrm{kg})$ in the type III and IV reticulocytes, a high 
Flow cytometric analysis of erythropoietic abnormality.

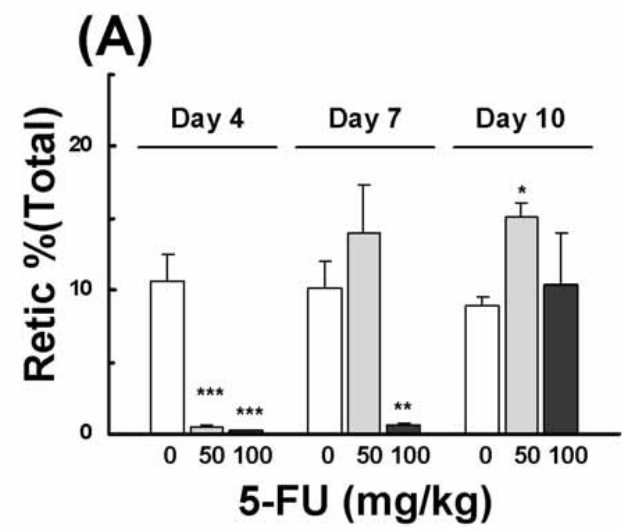

(B)

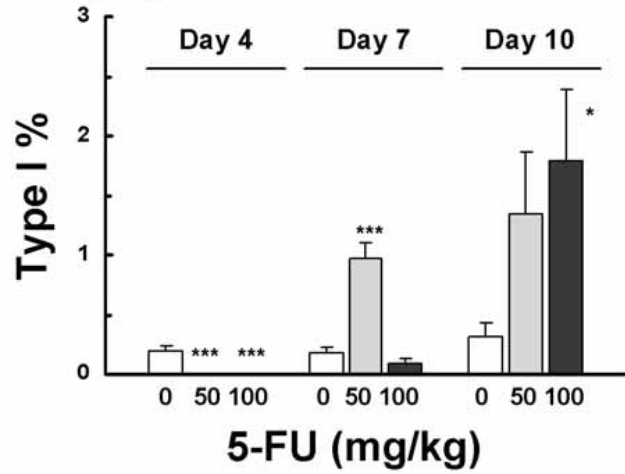

(C)

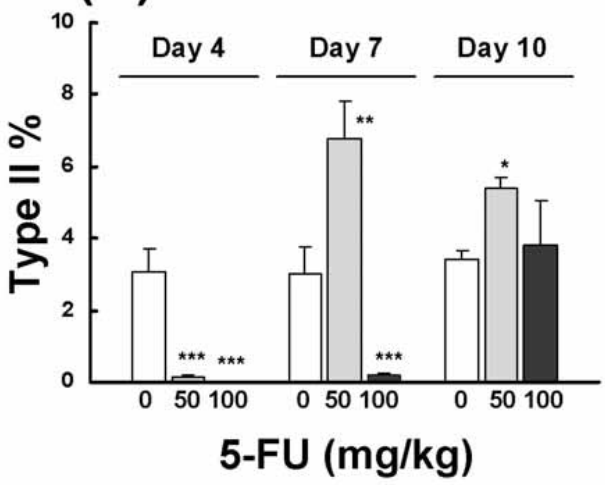

(D)

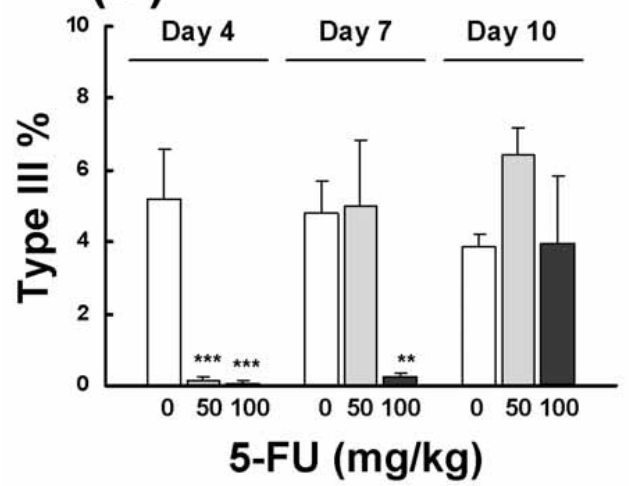

(E)

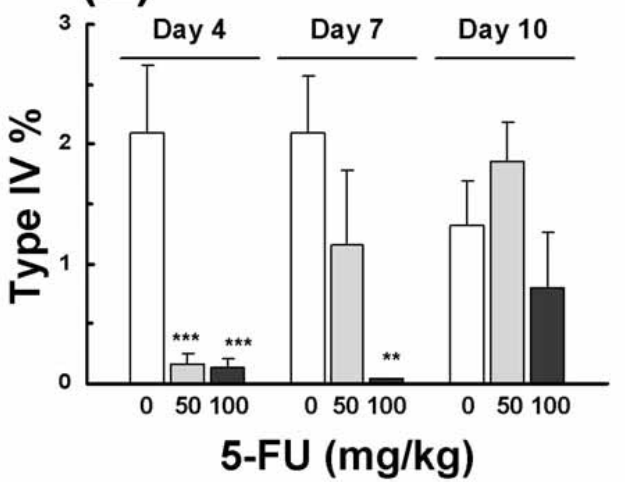

Fig. 4. Results of microscopic manual counting of peripheral blood collected from 5-fluorouraciladministered rats.

Peripheral blood was collected 4, 7 and 10 days after the administration of 5-fluorouracil (5FU) at 50 and $100 \mathrm{mg} / \mathrm{kg}$. Control animals $(0 \mathrm{mg} / \mathrm{kg})$ were administered physiological saline. Heilmyer's maturation groups were differentiated as follows: type I, the reticulum appears in the form of a dense clump; type II, appears in the form of a wreath; type III, the wreath has disintegrated; type IV, only a few scattered granules of the reticulum remain. Values are the means and $\operatorname{SD}(\mathrm{n}=3) . * \mathrm{p}<0.05, * * \mathrm{p}<0.01, * * * \mathrm{p}<0.001$ 
value as compared with the control was not observed.

\section{Changes in the maturity of reticulocytes after administration of APHZ}

Fig. 5 shows the reticulocyte ratio, CMI and RDI examined by EPICS XL-MCL using peripheral blood obtained from rats after two weeks of repeated oral administration of APHZ at 1 and $3 \mathrm{mg} / \mathrm{kg}$. A dosedependent increase was observed in the reticulocyte ratio, $\mathrm{CMI}$ and $\mathrm{RDI}$.

Table 1 shows results of blood chemical and hematological examinations, and the bone marrow examination/myelogram in rats after two weeks of repeated oral administration of APHZ. In the blood chemical examination, increases of total bilirubin and serum Fe were observed in APHZ-administered rats. In the hematological examination, dose-dependent decreases of the erythrocyte count, hemoglobin, hematocrit and MCHC were observed. In contrast to these parameters, increases of $\mathrm{MCH}$ and MCV were observed in APHZ-administered rats. In the bone marrow examination/myelogram, increases of bone marrow nucleated cells and the erythroblast ratio, and decrease of the $\mathrm{M} / \mathrm{E}$ ratio were observed. In addition to the results mentioned above, gross pathological findings and changes of organ weights in APHZ-administered rats were also observed as follows: at autopsy, a large spleen at 1 and $3 \mathrm{mg} / \mathrm{kg}$, dark reddish change in spleen at 1 and $3 \mathrm{mg} / \mathrm{kg}$, and in liver and kidney at 3 $\mathrm{mg} / \mathrm{kg}$; increase of spleen weight at 1 and $3 \mathrm{mg} / \mathrm{kg}$.

\section{DISCUSSION}

The enumeration of reticulocytes and examination of the maturity of reticulocytes are important hematological measurements for obtaining information about erythropoiesis. To improve reticulocyte measurements, an automated reticulocyte counting method using FCM has been developed (Koepke, 1991; Siekmeier et al., 2000), and its clinical value has been well studied. However, regarding non-clinical toxicity studies, the evaluation of reticulocyte maturity using the total reticulocyte count obtained by the FCM method has yet to be assessed. Therefore, we performed a FCM-based analysis of the maturity of reticulocytes with the total reticulocyte count using peripheral blood obtained from rats administered 5-FU or APHZ in the present study to clarify whether the FCM method would be useful in toxicity assessments, as well as clinical applications.

We had reported that EPICS-XL MCL equipped with a reticONE system showed good reproducibility and a correlation with conventional microscopic methods for examining Retic\% (Miura et al., 1999). How-
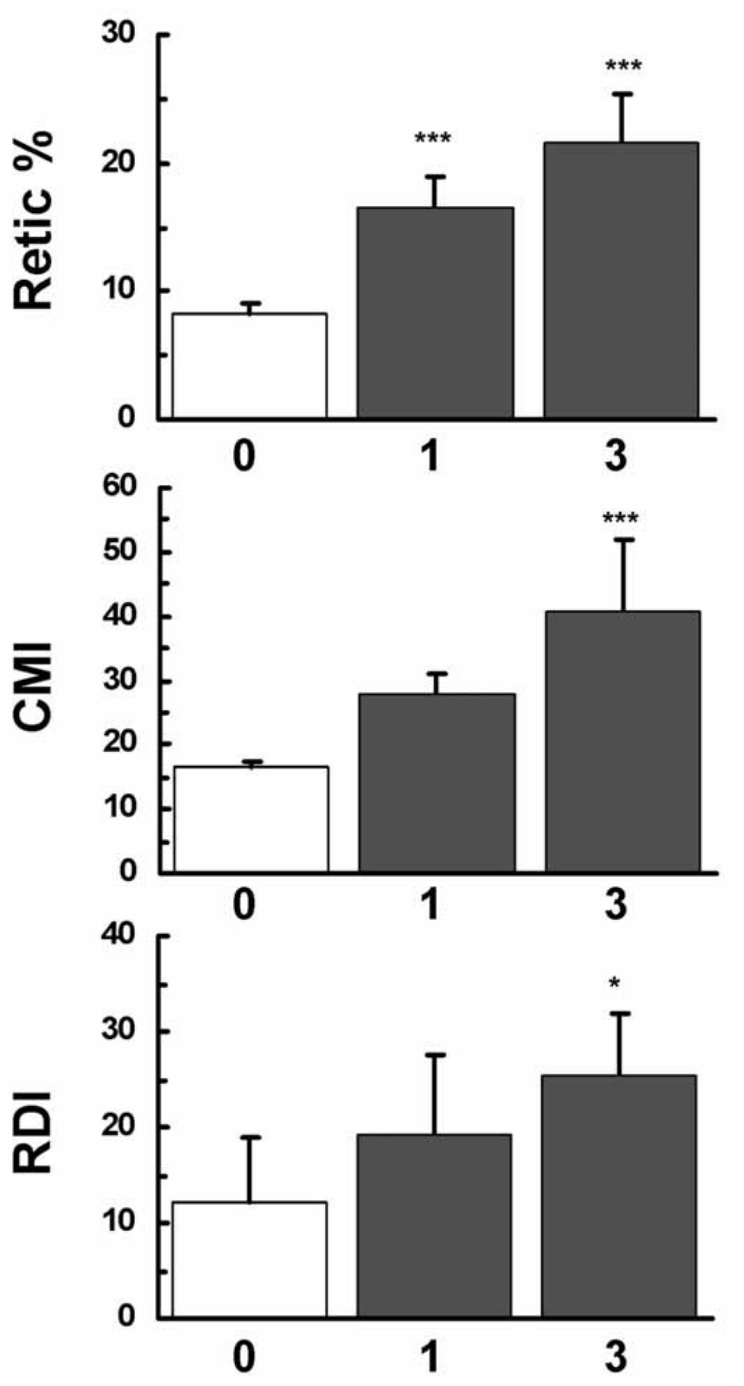

APHZ (mg/kg)

Fig. 5. The reticulocyte ratio (Retic\%), Cell Maturity Index (CMI) and Retic Distribution Index (RDI) of peripheral blood collected from acethylphenyl hydrazineadministered rats.

Peripheral blood was collected after 2 weeks of repeated oral administration of acethylphenyl hydrazine (APHZ) at 1 and $3 \mathrm{mg} / \mathrm{kg}$. Control animals $(0$ $\mathrm{mg} / \mathrm{kg}$ ) were administered distilled water. Retic\%, CMI and RDI were analyzed using EPICS XL-MCL. Values are the means and SD $(\mathrm{n}=5) . * \mathrm{p}<0.05, * * *$ $\mathrm{p}<0.001$ 
Flow cytometric analysis of erythropoietic abnormality.

ever, the value of CMI or RDI for assessing erythropoietic toxicity was not examined in the previous study. As shown in Fig. 2, changes in the CMI and RDI values were considered to well reflect the decrease (Fig. 2A) or increase (Fig. 2B) in the cellular RNA content of the reticulocyte population, as indicated by the distribution pattern in the histogram, and it was suggested that the maturity of reticulocytes could be evaluated using these parameters in rat peripheral blood. In addition, as shown in Fig. 3, changes in CMI and RDI after 5-FU administration were observed prior to Retic\% and clearly observed at the dose, whereas Retic\% was slightly affected. As for the correlation between the FCM and microscopic methods, the decrease or increase of CMI and RDI after the administration of 5FU at 50 or $100 \mathrm{mg} / \mathrm{kg}$ showed a good correlation with the changes in Heilmyer's type I and II (Fig. 4). These results indicated that the immature fraction of reticulocytes obtained by the FCM method could provide useful and early measurements of toxicity and recovery following chemical-induced erythropoietic toxicity.

Anemia is classified into two pathophysiological groups, hypoplastic anemia and hyperplastic anemia (Koepke, 1991). 5-FU is known as a myelosuppressive cytostatic agent, and more strongly affects cells with a high level of proliferating activity, including hematopoietic progenitor cells (Cheng et al., 2000). Considering the effect of 5-FU on bone marrow, the changes of CMI and RDI observed in the present study after the administration of 5-FU (Fig. 3) are considered to reflect the toxic response relating to hypoplastic anemia. APHZ was reported to induce hemolytic anemia (Benzi et al., 1964; Gewerc, 1972), and hemolytic anemia caused a great increase in reticulocytes, which means a hyperplastic response, in a clinical study (d'Onofrio et al., 1996). In the present study, as shown in Table 1, changes in the blood chemical (increases of total bilirubin and serum Fe) and hematological (decrease of erythrocyte count, hemoglobin, hematocrit and MCHC, and increase of $\mathrm{MCH}$ and $\mathrm{MCV}$ ) examinations were observed in APHZ-treated rats, in addition to the changes in reticulocyte analysis (dosedependent increase of CMI and RDI as shown in Fig. $5)$. In the bone marrow examination/myelogram, an increase in the bone marrow nucleated cells and erythroblast ratio, and decrease in the $\mathrm{M} / \mathrm{E}$ ratio were also observed. Furthermore, gross pathological findings (large spleen, dark reddish change in the spleen, liver and kidney) and increase of spleen weight were observed. The data mentioned above were considered

Table 1. Results of blood chemical and hematological examinations and the bone marrow examination/myelogram in acethylphenyl hydrazine-administered rats.

\begin{tabular}{|c|c|c|c|c|c|c|c|}
\hline \multirow[b]{2}{*}{$\begin{array}{c}\text { Dose } \\
(\mathrm{mg} / \mathrm{kg})\end{array}$} & & \multicolumn{2}{|c|}{ Blood chemistry } & \multicolumn{4}{|c|}{ Hematology } \\
\hline & & $\begin{array}{l}\text { Total bilirubin } \\
(\mathrm{mg} / \mathrm{dl})\end{array}$ & $\begin{array}{c}\mathrm{Fe} \\
(\mu \mathrm{g} / \mathrm{dl})\end{array}$ & $\begin{array}{c}\text { Erythrocytes } \\
\left(\times 10^{4} / \mu \mathrm{l}\right)\end{array}$ & $\begin{array}{l}\text { Hemoglobin } \\
(\mathrm{g} / \mathrm{dl})\end{array}$ & $\begin{array}{l}\text { Hematocrit } \\
(\%)\end{array}$ & $\begin{array}{l}\mathrm{MCHC} \\
(\mathrm{g} / \mathrm{dl})\end{array}$ \\
\hline \multirow[t]{2}{*}{0} & Mean & 0.03 & 156 & 837 & 16.0 & 47.9 & 33.4 \\
\hline & \pm S.D. & \pm 0.01 & \pm 27 & \pm 31 & \pm 0.5 & \pm 1.7 & \pm 0.5 \\
\hline \multirow[t]{2}{*}{1} & Mean & $0.09 * *$ & 190 & $663 * * *$ & $14.1 * * *$ & $42.2 * * *$ & 33.4 \\
\hline & \pm S.D. & \pm 0.02 & \pm 31 & \pm 64 & \pm 0.6 & \pm 2.2 & \pm 0.5 \\
\hline \multirow[t]{2}{*}{3} & Mean & $0.25 * * *$ & $341 * * *$ & $414 * * *$ & $11.3 * * *$ & $42.4 * * *$ & $26.6^{* * *}$ \\
\hline & \pm S.D. & \pm 0.04 & \pm 97 & \pm 20 & \pm 0.4 & \pm 1.2 & \pm 1.0 \\
\hline \multirow{2}{*}{\multicolumn{2}{|c|}{$\begin{array}{c}\text { Dose } \\
(\mathrm{mg} / \mathrm{kg})\end{array}$}} & \multicolumn{2}{|c|}{ Hematology } & \multicolumn{4}{|c|}{ Bone marrow examination/Myelogram } \\
\hline & & $\begin{array}{c}\mathrm{MCH} \\
(\mathrm{pg})\end{array}$ & $\begin{array}{l}\mathrm{MCV} \\
(\mathrm{fl})\end{array}$ & $\begin{array}{c}\text { Bone marrow nucleated } \\
\text { cells }\left(\times 10^{4} / \mu \mathrm{l}\right)\end{array}$ & \multicolumn{2}{|c|}{$\begin{array}{l}\text { Total erythroblasts } \\
(\%)\end{array}$} & $\mathrm{M} / \mathrm{E}$ ratio \\
\hline \multirow[t]{2}{*}{0} & Mean & 19.1 & 57.2 & 197 & \multicolumn{2}{|c|}{35.4} & 0.68 \\
\hline & \pm S.D. & \pm 0.6 & \pm 1.7 & \pm 30 & \multicolumn{2}{|c|}{ \pm 0.9} & \pm 0.08 \\
\hline \multirow[t]{2}{*}{1} & Mean & $21.3 * *$ & $63.9 *$ & $298 * *$ & \multicolumn{2}{|c|}{$59.9 *$} & $0.29 * *$ \\
\hline & \pm S.D. & \pm 1.5 & \pm 3.8 & \pm 14 & \multicolumn{2}{|c|}{ \pm 10.2} & \pm 0.09 \\
\hline \multirow[t]{2}{*}{3} & Mean & $27.2 * * *$ & $102.7 * * *$ & $292 * *$ & \multicolumn{2}{|c|}{$66.1 *$} & $0.23 * *$ \\
\hline & \pm S.D. & \pm 1.1 & \pm 6.5 & \pm 17 & \multicolumn{2}{|c|}{ \pm 14.7} & \pm 0.13 \\
\hline
\end{tabular}

Peripheral blood or bone marrow was collected after two weeks of repeated oral administration of acethylphenyl hydrazine at 1 and $3 \mathrm{mg} / \mathrm{kg}$. Control animals $(0 \mathrm{mg} / \mathrm{kg})$ were administered distilled water. The number of animals in each group was five for the blood chemical and hematological examinations, and three for the bone marrow examination/myelogram. $* \mathrm{p}<0.05, * * \mathrm{p}<0.01, * * *$ $\mathrm{p}<0.001$ 
as toxicological changes caused by increased loss or dysfunction of erythrocytes and a resulting increase in compensative erythropoiesis. It was strongly suggested that the clear increase in the immature reticulocyte fraction observed in the present study (Fig. 5) coincided with the enhancement of reticulocyte production caused by APHZ-induced hemolytic anemia. Based on these results, it was suggested that the reticulocyte maturity fraction (CMI and RDI) obtained by the FCM method would be useful and valuable to evaluate both hyper- and hypoplastic anemia in experimental animals, as well as humans.

We previously reported the myelotoxic effect after 5-FU administration in rats examined by FCM analysis on a lineage-specific cell surface antigen (Kakiuchi et al., 2004). In comparison with the timecourse (kinetics of decrease and recovery) after the administration of $50 \mathrm{mg} / \mathrm{kg}$ reported in the above paper, the changing pattern of CMI and RDI showed a good correlation with the changing pattern of erythroblast numbers in bone marrow. Retic\% change in peripheral blood showed a delayed pattern as compared with the change observed in bone marrow. These results indicated that the CMI and RDI would be more sensitive and rapid toxicological indicators, which reflect the changes in bone marrow.

In the present study, while statistically significant changes in the CMI and RDI were observed after the administration of $5-\mathrm{FU}$ at $10 \mathrm{mg} / \mathrm{kg}$ (Fig.3), clear changes were not observed in results of the microscopic method at this dose. Whereas the decrease or increase of CMI and RDI showed a good correlation with the changes in Heilmyer's type I and II (Fig. 4) at 50 or $100 \mathrm{mg} / \mathrm{kg}$, the data at $10 \mathrm{mg} / \mathrm{kg}$ seemed to indicate a discrepancy between the two methods. The reason for this phenomenon was considered to be as follows. First, the timing of the microscopic observation (4, 7 and Day 10) was chosen based on a peak point or trough of FCM analysis after the administration of 50 or $100 \mathrm{mg} / \mathrm{kg}$ to assess the correlation between the two methods, as clearly as possible. However, changes in the CMI and RDI at $10 \mathrm{mg} / \mathrm{kg}$ were observed earlier than these time points, and it would be suggested that the changes in Heilmyer's type I and II fraction may be observed on Day 2 or 3 at $10 \mathrm{mg} / \mathrm{kg}$. Second, the number of cells examined differed greatly between the two methods. With the FCM method, the number of erythrocytes analyzed was 40 times that with the microscopic method. Therefore, it would be suggested that this difference in the number of cells analyzed may affect the sensitivity of the method, especially when assessing a weak toxic effect. As mentioned in our previous report (Kakiuchi et al., 2004), the suppressive effect on erythropoiesis in bone marrow was observed after the administration of $5-\mathrm{FU}$ at $10 \mathrm{mg} / \mathrm{kg}$; therefore, this dose of 5-FU possibly possesses erythropoietic toxicity. Considering the fact mentioned above, any overestimation of toxicity by assessing CMI and RDI is likely to be minimal. However, careful consideration of the data of microscopic observations and bone marrow examinations would be important for applying the FCM method to an appropriate toxicological assessment.

As for the application of the FCM method to nonclinical studies, especially genotoxicity studies, Criswell et al. (1998) reported that the FCM methodology provided a more rapid and thorough approach to the assessment of bone marrow and spleen cytotoxicity than the conventional manual method of scoring the polychromatic erythrocyte/normochromatic erythrocyte ratio alone. Theirs was the first flow cytometric procedure to capitalize on the changes in fluorescence intensity within a bone marrow/spleen erythrocyte population to predict cytotoxicity in experimental animals. However, they did not examine this in detail from a physiopathological standpoint. Data of the present study, especially for the CMI and RDI as mentioned above, are considered to be important, because they are useful and valuable for assessing and predicting impairments of erythropoiesis by examining reticulocytes in peripheral blood.

Siekmeier et al. (2000) suggested that the automated reticulocyte counting method using FCM allows efficient and reliable determination of reticulocyte counts under routine clinical conditions. FCM methods allow the additional determination of the immature reticulocyte fraction, which allows further differentiation between "young" and "mature" reticulocytes. In principle, the enhanced production of reticulocytes causes an increase in the number of "young" reticulocytes, whereas the total number of reticulocytes is only moderately affected. In fact, measurement of the immature reticulocyte fraction further improved the diagnostic value of an automated reticulocyte count in clinical applications (Davis and Bigelow, 1990; Davis et al., 1993; Greinix et al., 1994; Spanish multicentric study group for hematopoietic recovery, 1994; Watanabe et al., 1994; Dalal et al., 1996; d'Onofrio et al., 1996). While the clinical value had been reported as mentioned above, regarding non-clinical toxicity studies, the value of the FCM method still needs to be clarified. The present study provides evidence that the 
Flow cytometric analysis of erythropoietic abnormality.

immature reticulocyte fraction is of toxicological value for early detection and monitoring, and could help with the diagnosis of hematological disorders in experimental animals, such as rats.

While the total peripheral erythrocyte number did not clearly change in the 5-FU-administered rats (data not shown), reticulocyte ratio, CMI and RDI values were once higher than the control level after decreasing and showing a trough, and then returning to the control level as shown in Fig. 3. As for the peripheral granulocyte, a similar temporarily high value and return to the control level was observed with the elevation of granulocyte colony stimulating factor and the following homeostatic regulation of myelopoiesis (Akamatsu et al., 1990). While the examination of changes in the levels of cytokines or biological mediators involved in the regulation of erythropoiesis is needed for further discussion, the changing pattern observed in the present study would reflect the biological response to the suppression of erythropoiesis by 5-FU, that is, stimulated erythropoiesis and the subsequent down regulation involving homeostasis of peripheral erythrocyte numbers. Al-Huniti et al. (2005) investigated changes in reticulocyte maturity using the FCM method following phlebotomy-induced stress erythropoiesis, and the relationship to the stimulated erythropoietin (EPO) in sheep. They reported a good relationship between EPO stimulation and the reticulocyte maturity index. Such a report is considered to support that the FCM assay in combination with EPO measurements would be a strong tool for examining the mechanism of erythropoietic toxicity.

In the present study, we performed a FCM analysis of the maturity of reticulocytes with the total reticulocyte count using peripheral blood obtained from rats given 5-FU or APHZ. In the hypoplastic anemia model (5-FU-administered rats), the decrease and recovery of the immature reticulocyte fraction was observed more rapidly as compared with the change in the total reticulocyte count, and sensitively regarding dose-dependency. In addition, there was good agreement between the microscopic results obtained by counting the Heilmyer's reticulocyte maturation groups and the immature fraction, CMI and RDI assessed by the FCM method after the administration of 50 and $100 \mathrm{mg} / \mathrm{kg} \mathrm{5-FU,} \mathrm{the} \mathrm{dose} \mathrm{at} \mathrm{which} \mathrm{clear}$ changes were obtained with the microscopic method. In the hyperplastic anemia model (hemolytic anemic rats after APHZ administration), a clear increase in the immature reticulocyte fraction coinciding with the enhancement of reticulocyte production was observed.
The results suggested that the automated FCM method could be a useful and valuable tool for assessing and predicting impairments of erythropoiesis, especially for the CMI and RDI.

\section{ACKNOWLEDGMENT}

The authors wish to sincerely thank Mr. H. Egawa, Beckman Coulter, for his expert technical support with the flow cytometer.

\section{REFERENCES}

Akamatu, K., Saito, H., Kamisango, K., Matsumoto, T., Endo, K., Ueno, K., Okawa, H., Oishi, T. and Koizumi, K. (1990): Therapeutic effect of recombinant human G-CSF on neutropenia caused by cancer chemotherapy in mice. Jpn. Pharmacol. Ther., 18 (Suppl. 9), 2487-2526.

Al-Huniti, N.H., Widness, J.A., Schmidt, R.L. and Veng-Pederse, P. (2005): Pharmacodynamic analysis of changes in reticulocyte subtype distribution in phlebotomy-induced stress erythropoiesis. J. Pharmacokinet. Pharmacodyn., 32, 359-376.

Benzi, G., Corona, G.L. and Frico, G.M. (1964): Effect of cobalt on anemia caused by acethylphenylhydrazine. I. Research in normal rats. Boll. Soc. Ital. Biol. Sper., 40, 1289-1292.

Brecher, G. and Schneiderman, M. (1950): A time saving device for the counting of reticulocytes. Am. J. Clin. Pathol., 20, 1079-1083.

Cheng, T., Rodrigues, N., Shen, H., Yang, Y., Dombkowski, D., Sykes, M. and Scadden, D.T. (2000): Hematopoietic stem cell quiescence maintained by p21cip1/waf1. Science, 287, 1804-1808.

Criswell, K.A., Krishna, G., Zielinski, D., Urda, G.A., Theiss, J.C., Juneau, P. and Bleavins, M.R. (1998): Use of acridine orange in flow cytometric evaluation of erythropoietic cytotoxicity. Mut. Res., 414, 49-61.

Dalal, B.I., Stockford, G.R., Naiman, S.C., Spinelli, J.J. and Phillips, G.L. (1996): Criteria for marrow engraftment: Comparison of reticulocyte maturity index with conventional parameters. Bone Marrow Transplantation, 17, 91-92.

Davis, B.H. and Bigelow, N. (1990): Clinical flow cytometric reticulocyte analysis. Pathobiology, 58, 99-106.

Davis, B.H., DiCorato, M., Bigelow, N. and 
Langweiler, M.H. (1993): Proposal for standardization of flow cytometric reticulocyte maturity index (RMI) measurements. Cytometry, 14, 318-326.

d'Onofrio, G., Kuse, R., Foures, C., Jou, J.M., Pradellas, M. and Zini, G. (1996): Reticuloytes in hematological disorders. Clin. Lab. Haem., 18, 29-34.

Dunnett, C.W. (1964): New tables for multiple comparisons with a control. Biometrics, 20, 482491.

Gewerc, J.C. (1972): Erythropoietic response to hemolytic and hemorrhagic anemia. Proc. Soc. Exp. Biol. Med., 139, 47-50.

Greinix, H.T., Linkesch, W., Keil, F., Kalhs, P., Schwarzinger, I., Schneider, B., Oesterreicher, C., Brugger, S., Kapiotis, S. and Lechner, K. (1994): Early detection of hematopoietic engraftment after bone marrow and peripheral blood stem cell transplantation by highly fluorescent reticulocyte count. Bone Marrow Transplantation, 14, 307-313.

Heilmeyer, L. and Westhäuser, R. (1932): Reifungs studien an überlebenden reticulozyten in vitro und ihre bedeutung für die schätzing der täglichen hemoglobin production in vivo. Z. Klin. Med., 121, 361-379.

Kakiuchi, S., Ohara, S., Ogata, S., Miura, D., Kasahara, Y. and Izawa,Y. (2004): Flow cyto- metric analyses on lineage-specific cell surface antigens of rat bone marrow to seek potential myelotoxic biomarkers: Status after repeated dose of 5-fluorouracil. J. Toxicol. Sci., 29, 101111.

Koepke, J.F. (1991): Reticulocyte counting. In Practical Laboratory Hematology (Koepke, J.A., ed.), pp. 99-107, Churchill Livingstone, New York.

Miura, D., Kobayashi, M., Iijima, T., Ogata, S., Yamana, K., Furukawa, J., Koike, Y. and Uno, H. (1999): Basic examination of reticulocytes count using the flow-cytometer (EPICS XL): A comparative study between flow-cytometer method and conventional method using hemolytic-anemia animals. J. Toxicol. Sci., 24, 306.

Siekmeier, R., Bierlich, A. and Jaro $\beta$, W. (2000): Determination of reticulocytes: Three methods compared. Clin. Chem. Lab. Med., 38, 245-249.

Spanish multicentric study group for hematopoietic recovery. (1994): Flow cytometric reticulocyte quantification in the evaluation of hematologic recovery. Eur. J. Haematol., 53, 293-297.

Watanabe, K., Kawai, Y., Takeuchi, K., Shimizu, N., Iri, H., Ikeda, Y. and Houwen, B. (1994): Reticulocyte maturity as an indicator for estimating qualitative abnormalities of erythropoiesis. J. Clin. Pathol., 47, 736-739. 